\title{
ARTICLE
}

\section{Isoalantolactone suppresses LPS-induced inflammation by inhibiting TRAF6 ubiquitination and alleviates acute lung injury}

Yun-he Ding ${ }^{1}$, Yun-duan Song ${ }^{2}$, Ya-xian $W^{1}{ }^{1}$, Hui-qiong He${ }^{1}$, Tian-hong $\mathrm{Yu}^{1}$, Yu-dong Hu${ }^{1}$, De-peng Zhang ${ }^{1}$, Hong-chao Jiang ${ }^{1}$, Kai-kai Yu ${ }^{1}$, Xiao-zong $\mathrm{Li}^{2}$, Lei Sun ${ }^{1}$ and Feng Qian ${ }^{1,3,4}$

Isoalantolactone (IAL) is a sesquiterpene lactone extracted from roots of Inula helenium $L$ and has shown anti-inflammatory effects. In this study we investigated the therapeutic effects of IAL on acute lung injury (ALI) and elucidated the mechanisms underlying its anti-inflammation potential in vitro and in vivo. Treatment with lipopolysaccharide (LPS, $100 \mathrm{ng} / \mathrm{mL}$ ) drastically stimulated production of inflammatory mediators such as NO, TNF- $a$, IL-1 $\beta$, and IL- 6 in mouse bone marrow-derived macrophages (BMDMs), which was dose-dependently suppressed by pretreatment with IAL $(2.5,5,10,20 \mu \mathrm{M})$. We further revealed that IAL suppressed LPSinduced NF-KB, ERK, and Akt activation. Moreover, the downregulation of non-degradable K63-linked polyubiquitination of TRAF6, an upstream transcription factor of NF-KB, contributed to the anti-inflammatory effects of IAL. ALI was induced in mice by intratracheal injection of LPS $(5 \mathrm{mg} / \mathrm{kg})$. Administration of IAL $(20 \mathrm{mg} / \mathrm{kg}$, i.p.) significantly suppressed pulmonary pathological changes, neutrophil infiltration, pulmonary permeability, and pro-inflammatory cytokine expression. Our results demonstrate that IAL is a potential therapeutic reagent against inflammation and ALI.

Keywords: isoalantolactone; lipopolysaccharide; TRAF6 ubiquitination; macrophage; acute lung injury

Acta Pharmacologica Sinica (2019) 40:64-74; https://doi.org/10.1038/s41401-018-0061-3

\section{INTRODUCTION}

Acute lung injury (ALI) is a life-threatening disease that causes high morbidity and mortality worldwide [1]. ALI is characterized by pulmonary inflammation, including injury of the alveolar epithelium and endothelium, lung edema, and infiltration of neutrophils [2-4]. Regarding less severe conditions, ALI can develop into acute respiratory distress syndrome (ARDS) [5]. As ALI progresses, it can lead to pulmonary complication, with ultimate deterioration of gas exchange and respiratory failure [6]. Therefore, alleviating ALI in the early stage is vital to control respiratory symptoms. It is well known that inflammation is closely associated with ALI [7]. Although various studies have been performed concerning the pathophysiology of ALI in the past decade, few effective methods or medicines have been identified to treat ALI [8]. Consequently, effective drugs and therapies are urgently required.

Lipopolysaccharide (LPS), the major component of the gramnegative bacterial cell wall, can induce the production of pro-inflammatory cytokines and activate various types of cells, including macrophages, epithelial cells, and endothelial cells [9-11]. LPS components are thought to have an essential role in initiating the inflammatory courses, resulting in ALI [12]. The
LPS-induced ALI animal model has been established to explore the mechanism and potential therapies of ALI [13].

After stimulation, LPS binds to its receptor, Toll-like receptor (TLR) 4, followed by the recruitment of downstream adaptors, such as MyD88 (myeloid differentiation primary response gene 88), tumor necrosis factor (TNF) receptor-associated factor 6 (TRAF6), and transforming growth factor- $\beta$-activated kinase 1 (TAK1) [14]. TRAF6 is the signaling transducer for both the TNF receptor superfamily and interleukin (IL)-1R/TLR superfamily [15]. TRAF6 delivers the signaling through ubiquitination and interaction with TAK1 [16], which activates the downstream mitogen-activated protein kinases (MAPKs) and nuclear factor-KB (NF-KB) signaling pathway [17]. TRAF6 contains ring finger domains that are commonly found in ubiquitin ligases (E3) [18]. Ubiquitin itself has seven lysine residues (K6, K11, K27, K29, K33, K48, and K63), each of which can be conjugated to another molecule to form different polyubiquitin chains. The classical ubiquitin-proteasome system is polyubiquitin chains linked through lysine 48 (K48) ubiquitin for degradation. However, some polyubiquitin species are not linked to K48. Among these species, the most abundant is non-degradable K63-linked polyubiquitin chains [19]. K63 polyubiquitination has an essential role in kinase activation in TLR

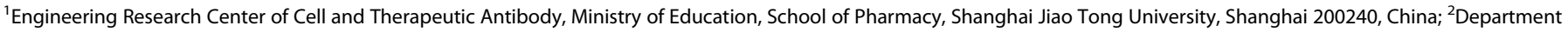

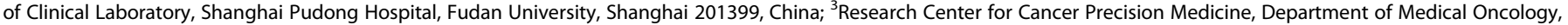

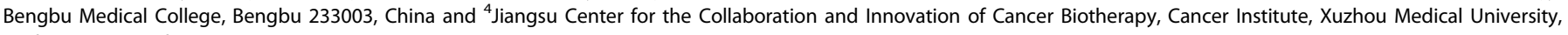
Xuzhou 221004, China

Correspondence: Feng Qian (fengqian@sjtu.edu.cn)

These authors contributed equally: Yun-he Ding, Yun-duan Song, Ya-xian Wu.

Received: 4 March 2018 Accepted: 5 June 2018

Published online: 16 July 2018 
pathways [20]. MAPKs are a superfamily composed of extracellular signal regulated kinase (ERK), c-jun N-terminal kinase (JNK), and p38 MAPK, which are critical in the regulation of inflammation and apoptosis [21, 22]. With LPS stimulation, NF-KB is translocated from the cytoplasm to the nucleus and is activated to cause the transcription of pro-inflammatory genes [23]. In addition, phosphoinositide 3 kinase (PI3K)/AKT, an essential downstream effector of TLR4 signaling, is involved in NF-KB activation [24].

Chinese herbal medicine has been used to treat human diseases clinically [25]. Sesquiterpene lactones possess many biological activities, including anti-proliferative, anti-inflammatory, and mycobacterial activities [26, 27]. Isoalantolactone (IAL), a sesquiterpene lactone, is extracted from the roots of Inula helenium L. Studies have suggested that IAL has anti-allergic, anti-oxidant [28], and anti-cancer activities during apoptosis related to the reactive oxygen species-mediated pathway [29]. IAL was reported to improve survival in a sepsis model and exhibit anti-inflammatory activity [30]. However, the pharmacological effect of IAL on $A L I$ and mechanism remain unclear.

In the present study, we evaluated the effects of IAL on LPSinduced lung injury. We found that IAL has anti-inflammatory effects both in vitro and in vivo. Moreover, the ubiquitination of TRAF6 was suppressed by IAL treatment. Our results suggested that IAL might be a potential drug to treat bacterial-induced infection and inflammation.

\section{MATERIALS AND METHODS}

Materials and reagents

IAL $\left(\mathrm{C}_{15} \mathrm{H}_{20} \mathrm{O}_{2}\right.$; MW, 232.31; purity >98\%) was purchased from TargetMol Co., Ltd (Shanghai, China). Dulbecco's modified Eagle's medium (DMEM), antibiotics (10000 units $/ \mathrm{mL}$ penicillin and $10000 \mu \mathrm{g} / \mathrm{mL}$ streptomycin), $0.25 \%$ trypsin, and phosphatebuffered saline (PBS) were purchased from Life Technologies (Grand Island, NY, USA). Fetal bovine serum (FBS) was purchased from EVERY GREEN by Zhejiang Tianhang Biotechnology Co., Ltd (Zhejiang, China). Recombinant murine macrophage colonystimulating factor (M-CSF) was purchased from PeproTech (Rocky Hill, NJ). The bicinchoninic acid (BCA) protein assay kit, nitric oxide (NO) assay kit (Griess reagent), and phenylmethylsulfonyl fluoride (PMSF) were purchased from Beyotime Biotechnology (Shanghai, China). The ReverTra Ace qPCR RT Kit was purchased from Toyobo (Osaka, Japan). LPS, DMSO, and protease inhibitor cocktail were obtained from Sigma (St Louis, MO, USA). The western blotting antibodies, including anti-p-p38, anti-p38, anti-p-JNK1/2, antiJNK1/2, anti-p-ERK, anti-ERK, anti-p-AKT, anti-AKT, anti-p-p65, antip65, anti- $\beta$-actin, anti-Myc-Tag, and anti-HA-Tag antibodies, were obtained from Cell Signaling Technology (Danvers, MA, USA). Antibodies to detect inhibitor of NF-KB (IKB) a and inducible NO synthase (iNOS) were obtained from Santa Cruz Biotechnology (California, USA). Horseradish peroxidase-conjugated goat antirabbit IgG antibody was obtained from Mei5 Biotechnology (Beijing, China). Fluorescein isothiocyanate (FITC)-conjugated antimouse Ly-6G (Gr-1) antibody and the enhanced chemiluminescence (ECL) kit were purchased from eBioscience by Thermo Fisher Scientific (Grand Island, NY, USA). RIPA Lysis Buffer was purchased from GenStar (Beijing, China). Protein-G Agarose beads were purchased from Millipore (Billerica, MA, USA). Enzyme-linked immunosorbent assay (ELISA) kits for mouse TNF- $\alpha$, IL-1 $\beta$, and IL- 6 were purchased from R\&D Systems (Minneapolis, MN, USA).

Cell isolation and cell culture

The bone marrow was collected from the femurs of C57/BL6 mice and was cultured in DMEM supplemented with 10\% FBS, a $1 \%$ penicillin/streptomycin mixture, and $10 \mathrm{ng} / \mathrm{mL}$ of M-CSF for 7 days $\left(37^{\circ} \mathrm{C}, 5 \% \mathrm{CO}_{2}\right)$. The medium was changed once. Bone marrowderived macrophages (BMDMs) were then collected and washed with PBS. After collection, BMDMs were incubated in DMEM supplemented with $10 \%$ FBS and the $1 \%$ penicillin/streptomycin mixture $\left(37^{\circ} \mathrm{C}, 5 \% \mathrm{CO}_{2}\right)$. HEK293 cells were purchased from ATCC (Manassas, VA) and were cultured in DMEM supplemented with $10 \%$ $\mathrm{FBS}$ and the $1 \%$ penicillin/streptomycin mixture $\left(37^{\circ} \mathrm{C}, 5 \% \mathrm{CO}_{2}\right)$.

Quantitative determination of the nitrite levels

The Griess reagent was used to determine the nitrite levels. BMDMs were pre-treated with IAL at $0,2.5,5,10$, and $20 \mu \mathrm{M}$ for $30 \mathrm{~min}$ before stimulation with LPS $(100 \mathrm{ng} / \mathrm{mL})$ for $12 \mathrm{~h}$. The cell culture supernatant was collected, and the Griess reagent was added. The NO levels were measured using a microplate reader (Flex Station 3; Molecular Devices, USA) at the wavelength of $540 \mathrm{~nm}$.

Western blotting analysis

Whole-cell lysates were prepared using loading buffer. The lung tissues were homogenized and lysed with RIPA Lysis Buffer plus PMSF. The proteins were separated using SDS-polyacrylamide gel electrophoresis and were transferred onto nitrocellulose membranes for antibody detection. The membranes were incubated with primary antibody overnight at $4{ }^{\circ} \mathrm{C}$ and then with secondary antibody for $2 \mathrm{~h}$ at room temperature. After washing, the signals were detected using an ECL kit. Quantitative analysis was accomplished using ImageJ software (National Institute of Mental Health, Bethesda, MD, USA).

Reverse-transcription PCR

BMDMs were stimulated with vehicle or $100 \mathrm{ng} / \mathrm{mL}$ of LPS. Total RNA was isolated from the cells or homogenized lung tissues using TRIzol Reagent and was reverse transcribed into cDNA using the ReverTra Ace qPCR RT Kit. For the quantification of CDNA, a StepOne Plus system (Thermo Fisher Scientific, Waltham, MA, USA) was applied for real-time PCR.

The following primers were used:

TNF-a, forward 5'-TTCTCATTCCTGCTTGTGG-3' and reverse 5'-ACTTGGTGGTTTGCTACG-3';

IL-1 $\beta$, forward 5'-CCAGCTTCAAATCTCACAGCAG-3' and reverse 5'-CTTCTITGGGTATTGCTTGGGATC-3';

IL-6, forward 5'-CCACCAAGAACGATAGTCAA- $3^{\prime}$ and reverse 5'-TTCCACGATTTCCCAGA-3';

GAPDH, forward 5'-TGCGACTTCAACAGCAACTC- $3^{\prime}$ and reverse 5'-CTTGCTCAGTGTCCTTGCTG-3'.

Enzyme-linked immunosorbent assay

BMDMs were pre-treated with IAL at $0,2.5,5,10$, and $20 \mu \mathrm{M}$ for $30 \mathrm{~min}$ before stimulation with LPS $(100 \mathrm{ng} / \mathrm{mL})$ for $4 \mathrm{~h}$. The supernatant was collected and the production of TNF-a, IL-1 $\beta$, and IL- 6 was measured by using ELISA kits. The concentrations of TNFa, IL-1 $\beta$, and IL-6 in bronchoalveolar lavage fluid (BALF) were also determined according to the manufacturer's instructions.

\section{Luciferase reporter assay}

HEK293 cells were seeded in 24-well plates and were transfected with $0.5 \mu \mathrm{g}$ of NF-kB luciferase reporter plasmid, $2.5 \mu \mathrm{g}$ of vector DNA or plasmid DNA (transforming growth factor $\beta$-activated kinase binding protein-3 [TAB3], p65, or TRAF6) using $6 \mu \mathrm{g}$ of polyethyleneimine on the following day according to the manufacturer's instructions. Eighteen hours after transfection, IAL $(10 \mu \mathrm{M})$ was added and the luciferase activity was determined $6 \mathrm{~h}$ later. The promoter activity was analyzed using a Luciferase Reporter Assay Kit.

Immunoprecipitation

HEK293 cells were co-transfected with HA-Ubiquitin (HA-Ub) and Myc-TRAF6. After $42 \mathrm{~h}$ of transfection, IAL $(10 \mu \mathrm{M})$ was added. After another $6 \mathrm{~h}$, the cells were washed with cold PBS and lysed on ice with RIPA lysis buffer containing protease inhibitor cocktail. Cell lysates were extracted and a portion was used as input for the 
loading control. The remaining lysate was incubated with anti-Myc antibody for $5 \mathrm{~h}$. Protein-G beads were subsequently added and shaken overnight at $4{ }^{\circ} \mathrm{C}$. The beads were then eluted with PBS and the protein was released by adding loading buffer. Immunoprecipitated sample and total cell lysate fractions were analyzed by immunoblotting.

In vivo ALI model

The 6- to 8-week-old male C57/BL6 mice $(20 \pm 3 \mathrm{~g})$ were purchased from Slac Laboratory Animal Corporation (Shanghai, China). After acclimation for several days under a specific pathogen-free environment $\left(25^{\circ} \mathrm{C}, 55 \%\right.$ humidity, with adequate food and water), the mice were divided randomly into 3 groups $(n=5)$. IAL $(20 \mathrm{mg} / \mathrm{kg})$ or solvent (castor oil: ethanol: physiological saline $=1: 1: 8$ ) was intraperitoneally injected $13 \mathrm{~h}$ or $1 \mathrm{~h}$, respectively, before the intratracheal administration of 5 $\mathrm{mg} / \mathrm{kg}$ LPS (intraperitoneal injection of $50 \mathrm{mg} / \mathrm{kg}$ pentobarbital sodium for anesthesia). The mice were killed $6 \mathrm{~h}$ after LPS administration.

To detect the therapeutic role of IAL in ALI, we treated the mice with IAL $(20 \mathrm{mg} / \mathrm{kg})$ or solvent (castor oil: ethanol: physiological saline $=1: 1: 8) 1 \mathrm{~h}$ after intratracheal administration of $5 \mathrm{mg} / \mathrm{kg}$ LPS. The mice were killed $6 \mathrm{~h}$ or $24 \mathrm{~h}$ after LPS administration.

Lung tissues and BALF were collected. The left lower lung was entirely fixed with $4 \%$ paraformaldehyde and the remaining lung lobes were frozen at $-80^{\circ} \mathrm{C}$. All experimental procedures described in this study were approved by the Animal Care and Use Committee of Shanghai Jiao Tong University. The animal experiments were performed in accordance with the National Institutes of Health Guide for the Care and Use of Laboratory Animals.

\section{Histological evaluation}

The fixed lung was dehydrated, embedded in paraffin and then sectioned into $5 \mu \mathrm{m}$ slicesusing a microtome (RM2235; Leica Biosystems, Wetzlar, Germany). Pathological changes were observed using a light microscope (RX51; Olympus Optical Co. Ltd, Tokyo, Japan) at $\times 200$ magnification after hematoxylin and eosin (H\&E) staining.

\section{Myeloperoxidase activity assay}

Neutrophil accumulation was evaluated by myeloperoxidase (MPO) activity. The lung tissue was homogenized and suspended in $0.5 \%$ hexadecyl trimethyl ammonium bromide (HTAB) diluted in $50 \mathrm{mM}$ phosphate buffer. After centrifugation, the pellet was resuspended in $0.5 \% \mathrm{HTAB}$ and the suspension was treated with a freeze-thaw procedure twice. The supernatant was collected and the protein concentration was determined using the BCA protein quantitation kit. Next, 3,3',5,5'-tetramethylbenzidine and $\mathrm{H}_{2} \mathrm{O}_{2}$ were added to the supernatant, followed by monitoring of the absorbance changes at $655 \mathrm{~nm}$ in $5 \mathrm{~min}$.

\section{BALF analysis}

The BALF was centrifuged and the red blood cells were lysed using lysis buffer followed by centrifugation. The remaining cells were washed, resuspended with PBS and counted. FITCconjugated anti-mouse Ly-6G (Gr-1) antibody was added to label the neutrophils. The samples were analyzed using a FACSCanto flow cytometer (Becton Dickinson). FlowJo 7.6 was used to analyze the data.

\section{Statistical analysis}

Graphpad 5.0 was used to generate all the graphs and statistical analysis. The values were presented as mean \pm SEM for at least three independent experiments. One-way analysis of variance followed by the Bonferroni $t$-test was used to compare the data among multiple groups. $P<0.05$ was considered significant.

\section{RESULTS}

IAL suppresses the expression of iNOS and inhibits NO production in BMDMs

The chemical structure of IAL is shown in Fig. 1a. To evaluate the effects of IAL in LPS-induced inflammatory responses in vitro, BMDMs were treated with LPS and IAL with the indicated concentrations for $12 \mathrm{~h}$, and released NO was measured. As expected, LPS drastically induced NO secretion from BMDMs into the medium. Treatment of IAL inhibited NO production in a dosedependent manner, with concentrations ranging from 2.5 to $20 \mu \mathrm{M}$ (Fig. 1b).

Next, we checked the expression of iNOS, which is responsible for NO production in cells. The data obtained from western blotting showed that pretreatment with $\operatorname{IAL}(0,2.5,5,10$, and $20 \mu \mathrm{M})$ inhibited LPS-induced iNOS expression in BMDMs in a dose-dependent manner (Fig. 1C). IAL at concentrations of 5, 10, and $20 \mu \mathrm{M}$ showed significant inhibition effects $(P<0.05$, Fig. $1 \mathrm{~d})$. These results suggested that IAL could alleviate inflammation via suppressing iNOS expression, thus reducing NO production.

IAL inhibits LPS-induced expression of pro-inflammatory cytokines in BMDMs

To investigate whether IAL affects the production of cytokines related to inflammation in BMDMs, quantitative RT-PCR was performed to check the mRNA expression of pro-inflammatory cytokines TNF- $\alpha$, IL-1 $\beta$, and IL- 6 in BMDMs. The TNF- $\alpha$, IL-1 $\beta$, and IL-6 levels were obviously increased upon LPS treatment compared with those of the control group. The results showed that pretreatment with IAL suppressed the mRNA levels of all three cytokines stimulated by LPS $(P<0.05$, Fig. 2a, c, e). Similarly, the protein levels of TNF- $a$, IL-1 $\beta$, and IL- 6 in BMDMs were reduced upon co-treatment with IAL and LPS in a dose-dependent manner $(P<0.05$, Fig. 2b, d, f).

IAL inhibits NF-KB activation and downregulates the MAPK and Akt signaling pathways in BMDMs

It is well established that NF-KB, MAPKs, and Akt have important roles in the inflammation process. Therefore, NF-KB activation was checked by detecting $\mathrm{p} 65$ phosphorylation and IKBa expression. MAPK activation was determined by detecting the phosphorylation of p38, JNK, and ERK, whereas the Akt/PI3K signaling pathway was analyzed by checking the phosphorylation of Akt in BMDMs. Our results demonstrated that IAL dose-dependently decreased p65 phosphorylation and increased IKBa expression induced by LPS, suggesting the downregulation of IAL concerning NF-KB activity (Fig. 3). When checking the MAPK pathway, only ERK phosphorylation was decreased by IAL treatment (Fig. 3). IAL could also affect the Akt pathway by decreasing Akt phosphorylation (Fig.3). Altogether, IAL inhibited activation of the signaling transduction molecules, including NF-KB, ERK, and Akt, with the most significant inhibition in NF-KB activation.

IAL suppresses NF-KB activity via targeting TRAF6

To explore how IAL suppressed the NF-KB pathway, we transfected HEK293 cells with TAB3, p65 and TRAF6, three signaling molecules that regulate NF-KB activation. Transfected HEK293 cells were treated with different concentrations of IAL for $6 \mathrm{~h}$, and the luciferase activities were then measured. It was shown that treatment with $20 \mu \mathrm{M}$ IAL suppressed the activity of NF-KB luciferase reporter driven by TRAF6, but not by TAB3 and p65, suggesting that IAL suppressed the activation of the NF-KB pathway via TRAF6 (Fig. 4a).

Subsequently, we investigated how IAL inhibits TRAF6 expression. As ubiquitination is an important mechanism to regulate TRAF6 expression, we co-transfected HEK293 cells with Myc-TRAF6 and HA-Ub to induce TRAF6 polyubiquitination. An immunoprecipitation (IP) assay was performed to check the ubiquitination level using anti-myc antibody. The addition of $10 \mu \mathrm{M}$ IAL was 
a<smiles>C=C1C(=O)OC2(I)CC3(I)C(=C)CCC[C@]3(C)CC12I</smiles>

Isoalantolactone (IAL)

$\mathrm{C}_{15} \mathrm{H}_{20} \mathrm{O}_{2} \quad \mathrm{MW}, 232.31$

C

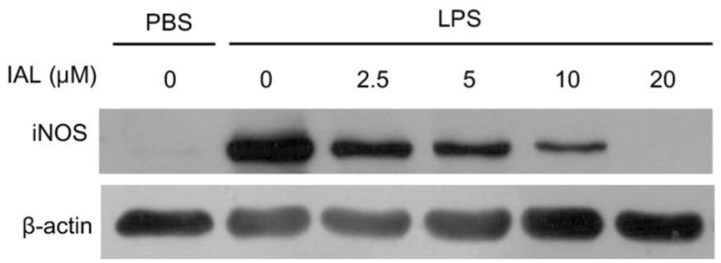

b
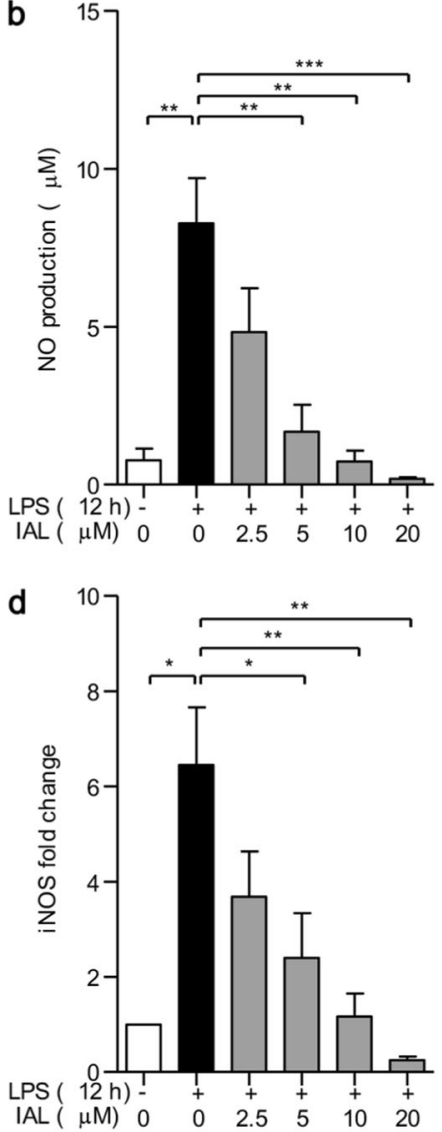

Fig. 1 Isoalantolactone inhibits NO production and suppresses iNOS expression in BMDMs. a Chemical structure of IAL. b NO production analysis of BMDM. c The protein expression of iNOS in BMDM was determined by western blotting. BMDMs were pre-treated with IAL at 0, 2.5, 5,10 , and $20 \mu \mathrm{M}$ for 30 min before stimulation with LPS $(100 \mathrm{ng} / \mathrm{mL})$ for $12 \mathrm{~h}$. The supernatant was collected for NO production analysis, and cell lysates were collected for Western blotting. $\beta$-Actin was used as a loading control. d Statistical analysis of iNOS expression in diagrams. The data represent the mean \pm SEM of three independent experiments $\left({ }^{*} P<0.05,{ }^{*} P<0.01\right.$, and $\left.{ }^{* * *} P<0.001\right)$

demonstrated to reduce TRAF6 ubiquitination ( $P<0.05$; Fig. $4 \mathrm{~b}, \mathrm{e})$. It was reported that seven lysine residues exist in ubiquitin, one of which is K63, the active site. Therefore, HEK293 cells were cotransfected with Myc-TRAF6 and HA-UbK63 and then were subjected to IP assay. The results revealed that K63-linked polyubiquitination of TRAF6 was reduced under treatment with $10 \mu \mathrm{M}$ IAL $(P<0.05$; Fig. $4 \mathrm{C}, \mathrm{f})$. We also found that IAL has no significant effect on the K48-linked polyubiquitination of TRAF6 (Fig. 4d, g). These results demonstrated that IAL negatively regulates the NF-KB pathway through hindering K63-linked polyubiquitination of TRAF6.

IAL attenuates LPS-induced ALI

The establishment of the ALI mouse model and IAL treatment procedure are described in "In vivo ALI model" and briefly shown in Fig. 5a. The pathological changes in lung tissues were analyzed by H\&E staining. After $6 \mathrm{~h}$ i.t. injection of LPS, lung tissue destruction was observed, including pulmonary edema, thickening of the alveolar wall, and inflammatory cell infiltration. On the other hand, treatment with $20 \mathrm{mg} / \mathrm{kg}$ of IAL effectively reduced the degree of lung injury (Fig. 5b). Subsequently, we detected the mRNA level of pro-inflammatory cytokines TNF- $\alpha$, IL-1 $\beta$, and IL- 6 in lung tissues and protein level of these cytokines in BALF using RT$P C R$ and ELISA, respectively. The results agreed with what we found in BMDMs challenged by LPS. IAL treatment significantly reduced the mRNA expression of TNF-a $(P<0.001$, Fig. $5 \mathrm{C})$, IL-1 $\beta$ $(P<0.05$, Fig. $5 \mathrm{~d})$, and IL-6 $(P<0.001$, Fig. $5 \mathrm{e})$, and similar results of the protein level in BALF were obtained $(P<0.001$, Fig. $5 f-h)$.
Moreover, the LPS-induced iNOS expression was decreased in lung tissues by IAL treatment (Fig. 5i, j). The phosphorylation of p65 was also reduced after IAL treatment (Figs. 5i, k). These data suggested that IAL not only inhibits inflammatory cytokine production and iNOS expression but also attenuates NF-KB signaling in vivo.

In addition, neutrophils in BALF were labeled with FITC-Gr-1 antibody and were counted by flow cytometry. LPS stimulation increased the percentage of neutrophils from $0.307 \%$ to $60.2 \%$, whereas IAL treatment decreased the percentage to $33.9 \%$ (Fig. 6a). The number of inflammatory cells in BALF was counted after red blood cell separation. LPS alone increased the inflammatory cell number in BALF, while the addition of $20 \mathrm{mg} /$ $\mathrm{kg}$ of IAL reduced the effect of LPS $(P<0.01$, Fig. $6 \mathrm{c})$. The accumulation of neutrophils was increased when challenged with LPS. Pretreatment with IAL $(20 \mathrm{mg} / \mathrm{kg})$ significantly decreased the percentage $(P<0.01$, Fig. $6 \mathrm{~b})$ and number of neutrophils $(P<0.01$, Fig. $6 \mathrm{~d}$ ). Next, MPO activity analysis was performed to determine neutrophil infiltration. Obviously, IAL treatment attenuated neutrophil infiltration caused by LPS challenge $(P<0.001$, Fig. $6 \mathrm{e})$. The total protein concentration in BALF, indicating the pulmonary permeability, was also measured. Consistent with the data of MPO activity analysis, the LPS-increased protein concentration was remarkably inhibited by IAL treatment $(P<0.05$, Fig. $6 f)$.

To evaluate the therapeutic role of IAL in ALI, we carried out an in vivo experiment in which mice were first challenged with LPS for $1 \mathrm{~h}$ and then were treated with IAL. Pulmonary permeability and neutrophil accumulation were measured 6 and $24 \mathrm{~h}$ after LPS 

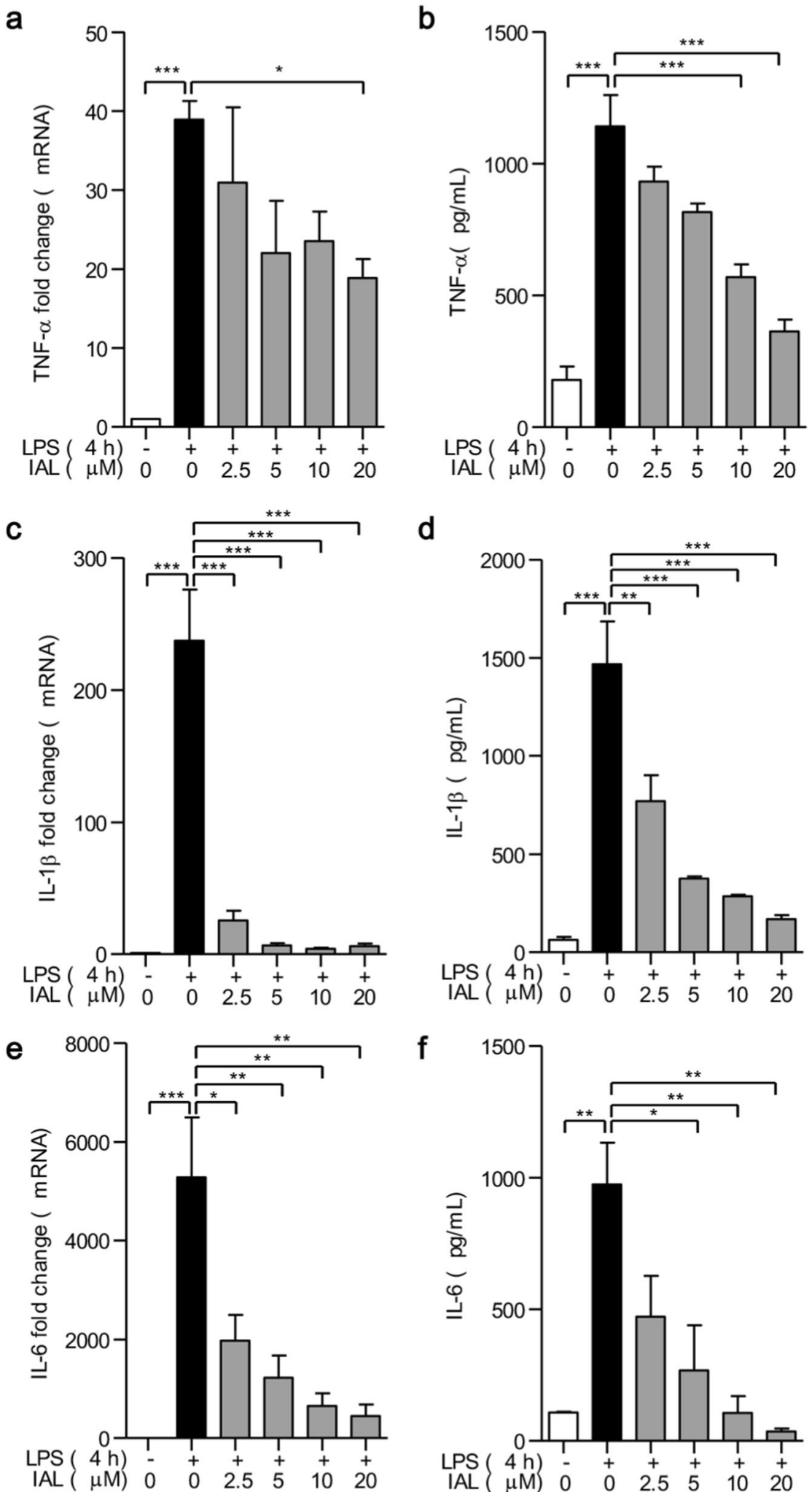

Fig. 2 IAL inhibits the LPS-induced expression of pro-inflammatory cytokines in BMDMs. The mRNA expression levels of a TNF- $\alpha$, $\mathbf{b}$ IL-1 $\beta$, and e IL-6 in BMDMs with the following treatments were evaluated by RT-PCR. The protein production levels of $\mathbf{b}$ TNF- $\alpha$, (d) IL-1 $\beta$ and $\mathbf{f}$ IL- 6 in supernatants were determined by ELISA. BMDMs were pre-treated with IAL at $0,2.5,5,10$, and $20 \mu \mathrm{M}$ for 30 min before stimulation with LPS $(100 \mathrm{ng} / \mathrm{mL})$ for $4 \mathrm{~h}$. The data represent the mean \pm SEM of three independent experiments $\left({ }^{*} P<0.05,{ }^{* *} P<0.01\right.$, and $\left.{ }^{* * * P} P<0.001\right)$ 


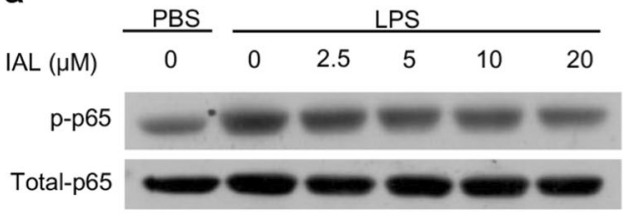

$\mathrm{I} \mathrm{BR}$

$\beta$-actin
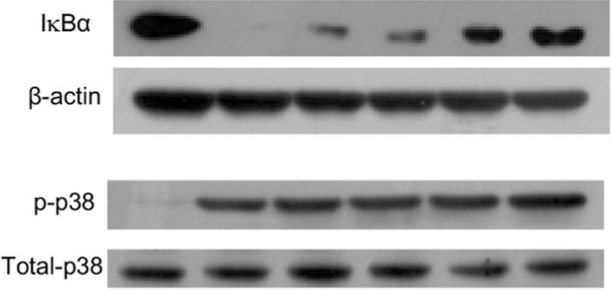

p-JNK

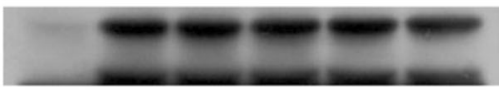

Total-JNK

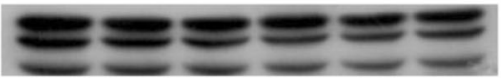

p-ERK

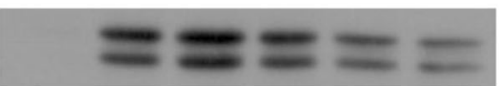

Total-ERK

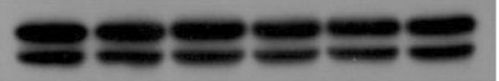

p-AKT

Total-AKT
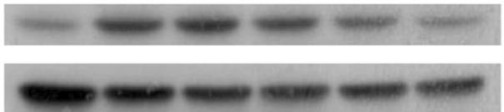

b
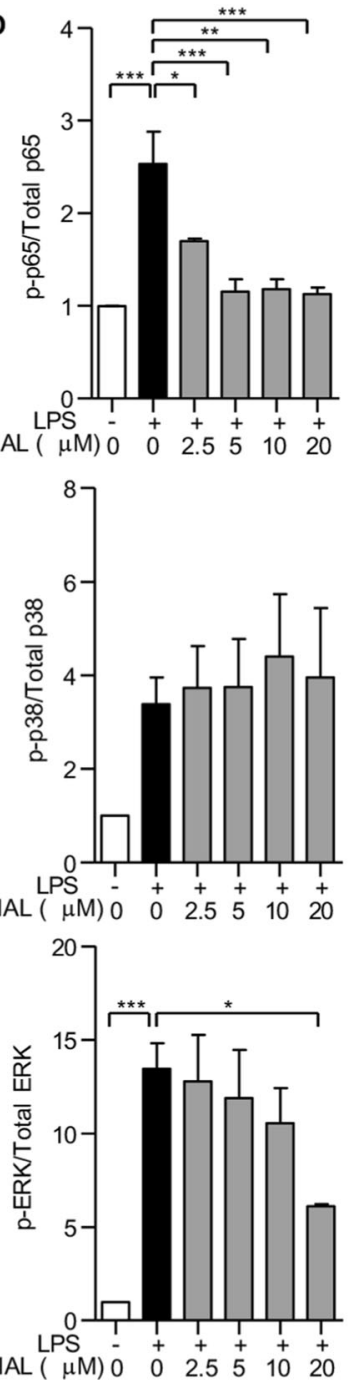
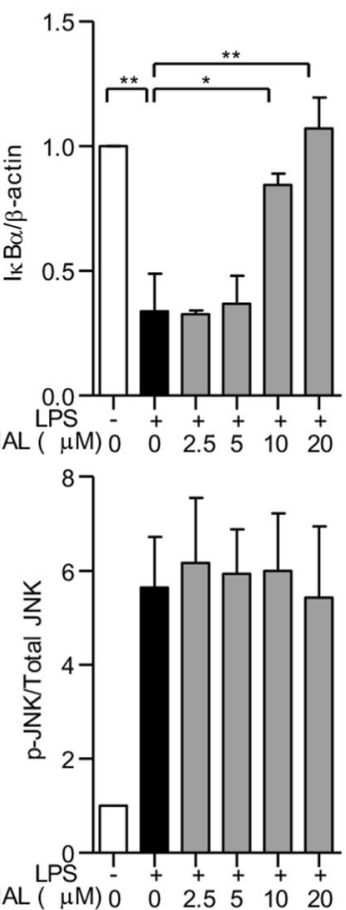

$\mathrm{AL}(\mu \mathrm{M}) 000025 \quad 5 \quad 10 \quad 20$

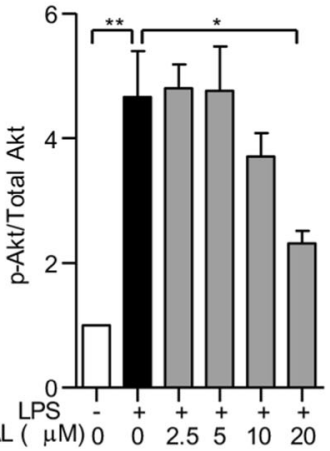

Fig. 3 IAL inhibits inflammation by deactivating NF- $\kappa B$, MAPKs, and the Akt signaling pathway in BMDMs. a The protein expression levels of p-p65, p65, IкB $\alpha, p-p 38, p 38, p-J N K, J N K, p-E R K, E R K, p-A K T$, and AKT were evaluated by western blotting. BMDMs were pre-treated with IAL at

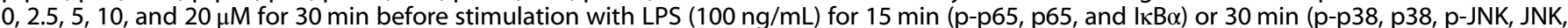
p-ERK, ERK, p-AKT, and AKT). $\beta$-Actin was used as a loading control. $\mathbf{b}$ Statistical analysis of the above assay in histograms. The data represent the mean \pm SEM of three independent experiments $\left({ }^{*} P<0.05,{ }^{* *} P<0.01\right.$, and $\left.{ }^{* * *} P<0.001\right)$

treatment. We treated the mice with IAL $(20 \mathrm{mg} / \mathrm{kg})$ or solvent $1 \mathrm{~h}$ after intratracheal administration of $5 \mathrm{mg} / \mathrm{kg}$ LPS. The mice were killed $6 \mathrm{~h}$ or $24 \mathrm{~h}$ after LPS administration. The procedure is briefly shown in Fig. 7a. The total protein concentration in BALF was elevated after LPS challenge for both $6 \mathrm{~h}$ and $24 \mathrm{~h}$, whereas the protein concentration in BALF was decreased in the IAL treatment group (Fig. 7b). The number of inflammatory cells in BALF was increased after LPS challenge for both $6 \mathrm{~h}$ and $24 \mathrm{~h}$. In response to IAL treatment, the amount of total inflammatory cells was reduced (Fig. 7c). Meanwhile, pulmonary neutrophil accumulation was also reduced with IAL treatment (Fig. 7d). All these results demonstrated that IAL has both protective and therapeutic effects against LPS-induced ALI.

\section{DISCUSSION}

Components of herbal medicines have been widely utilized to treat various diseases. It was reported that alantolactone, a sesquiterpene lactone extracted from I. helenium $L$, inhibited NO production, iNOS expression, and TNF-a by downregulating NF-KB and MAPK pathways in RAW264.7 cells [31], making alantolactone a potential therapeutic agent against inflammation [31]. IAL is another lactone isolated from I. helenium $L$ that shares a similar structure with alantolactone. It was reported that IAL could inhibit the production of NO and cytokines in peritoneal macrophages and RAW264.7 cells and protect mice from LPS-induced sepsis by inhibiting NF-KB activation in the liver [30]. However, the underlying mechanism remains unclear. In this study, we further expanded the understanding of the anti-inflammatory properties of IAL using in vivo LPS-induced ALI models and investigated the ubiquitination of TRAF6 to illustrate how IAL inactivates the NF-KB signaling pathway.

Inflammation is a mechanism to defend against exogenous pathogens and is involved in various physiological and pathological processes [32]. Various progressive diseases, including ALI, are associated with inflammation. LPS, the primary constituent of Gram-negative bacteria, is a ligand of TLR4 that induces inflammatory syndromes such as multiple organ injury and sepsis [33]. Macrophages have an important role in inflammatory responses via the secretion of pro-inflammatory cytokines and mediators [34]. When stimulated with LPS, macrophages have the capacity to produce high amounts of NO [35]. High levels of NO 
a

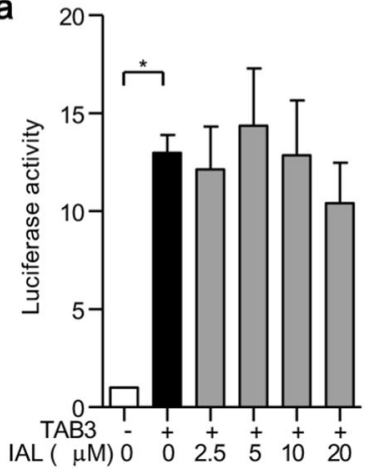

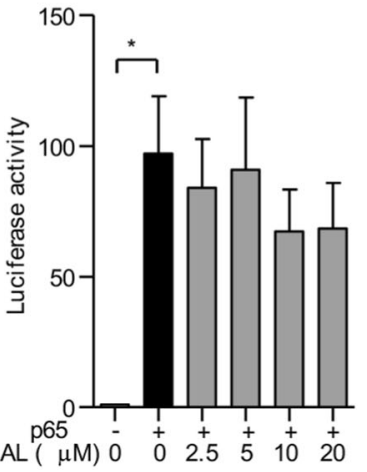

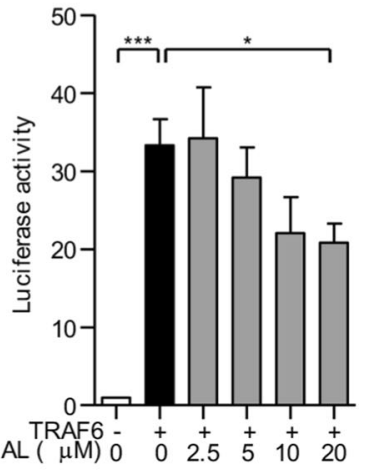

b

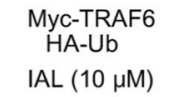

IP: Myc $\mid$\begin{tabular}{l|l} 
IB: HA \\
IB: Myc
\end{tabular}

Input IB: Myc

C

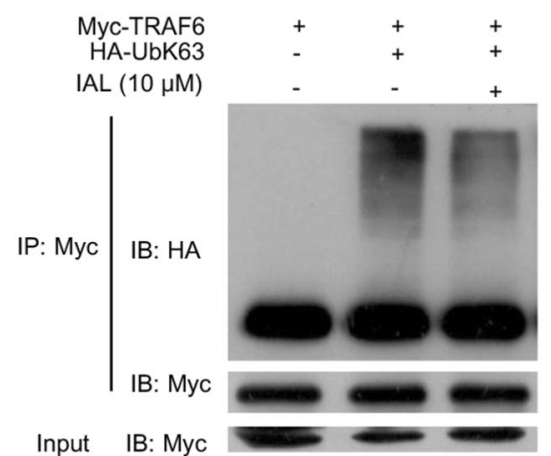

d

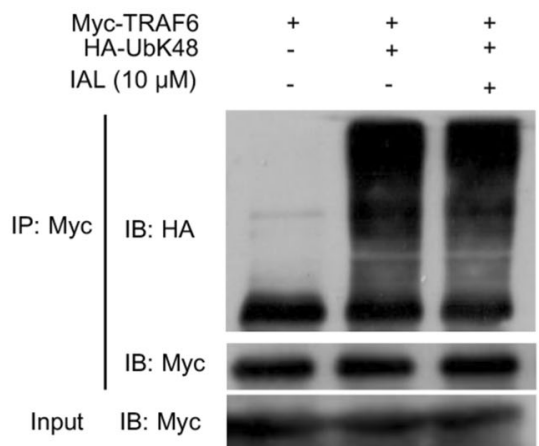

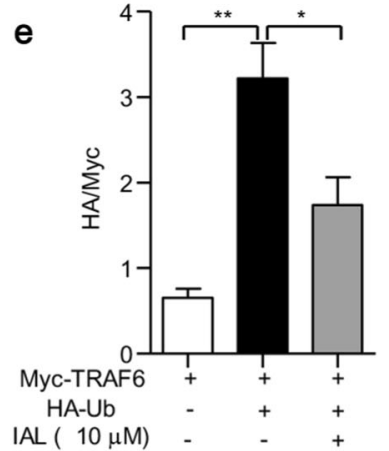

f

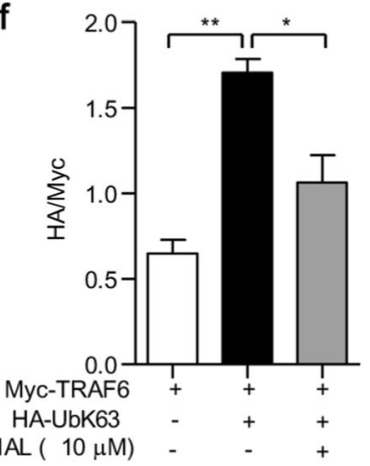

g

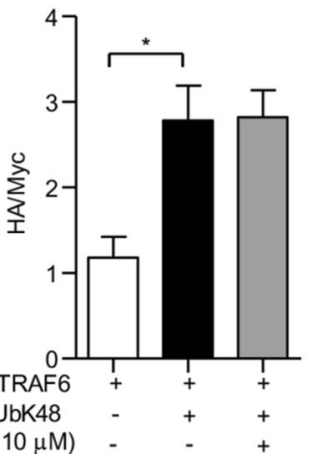

Fig. 4 Isoalantolactone suppresses the activation of the NF- $\mathrm{KB}$ pathway by downregulating TRAF6 ubiquitination. a HEK293 cells were transfected with TAB3, p65, or TRAF6 together with an NF-KB luciferase reporter plasmid. The cells were treated with or without IAL at different concentrations, and luciferase activity was measured. b-d The extent of TRAF6 ubiquitination in HEK293 cells transfected with MycTRAF6 alone, co-transfected with Myc-TRAF6, and b HA-Ub, c HA-UbK63, or (d) HA-UbK48 with or without $10 \mu \mathrm{M}$ IAL treatment was determined by immunoprecipitation. Cell lysates were subjected to IP with an anti-Myc Ab, followed by western blot analysis with an anti-HA or anti-Myc Ab. Total cell lysates were used as the control (Input). e-g Statistical analysis of TRAF6 ubiquitination in (b-d) histograms. The data represent the mean \pm SEM of three independent experiments $\left(* P<0.05,{ }^{* *} P<0.01\right.$, and $\left.{ }^{* * *} P<0.001\right)$

are produced in response to inflammatory stimuli and mediate pro-inflammatory and destructive effects caused mainly by iNOS [36]. In this study, we found that IAL inhibited NO production and that the expression of iNOS in LPS-stimulated BMDMs. Meanwhile, during inflammation, excessive pro-inflammatory cytokines are produced in macrophages and have the capacity to initiate and perpetuate the inflammatory response. Among them, the remarkable elevation of TNF- $\alpha, \mathrm{IL}-1 \beta$, and IL- 6 expression is 


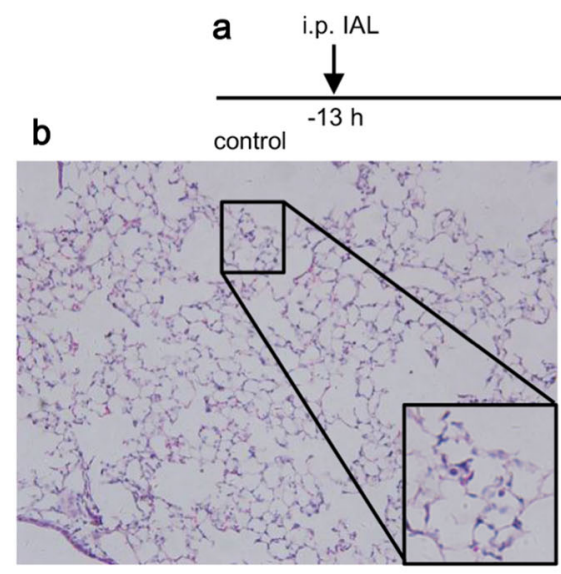

C

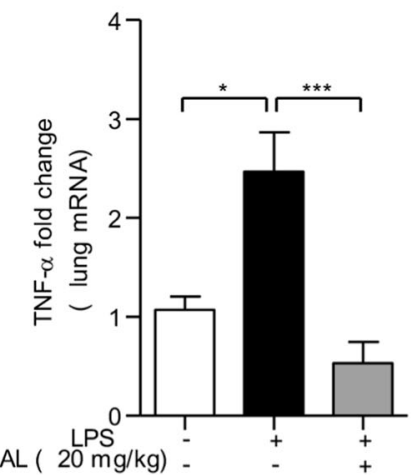

f

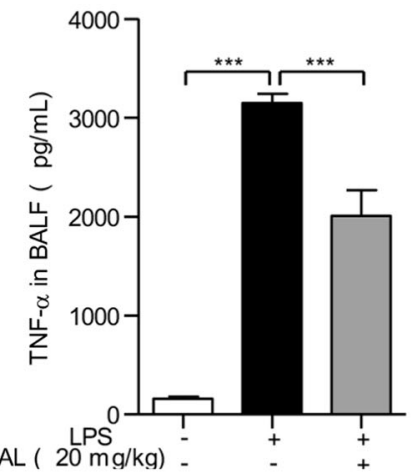

i

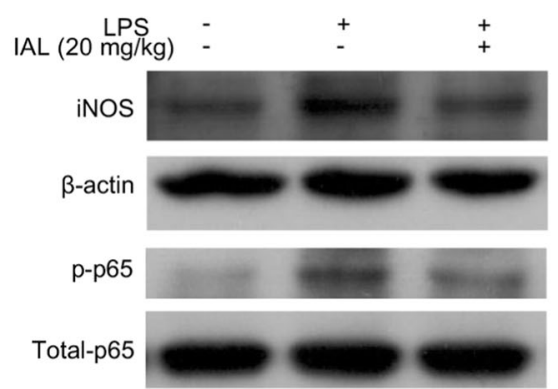

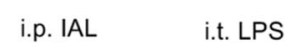

i.p. IAL i.t. LPS Analysis

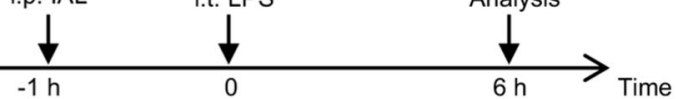

LPS + vehicle

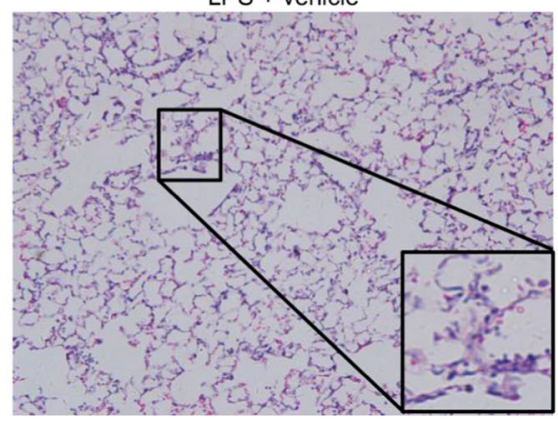

d

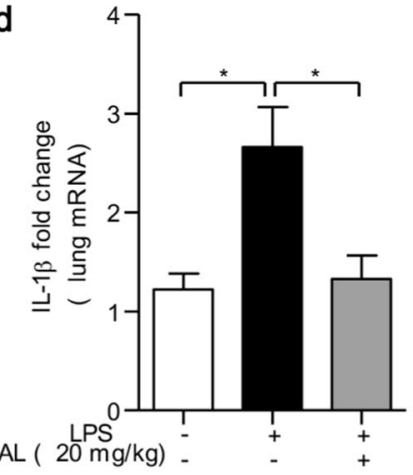

g

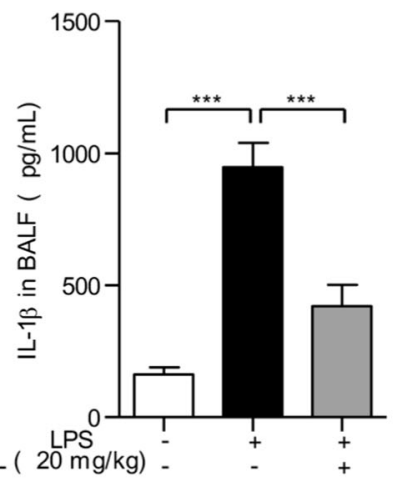

j

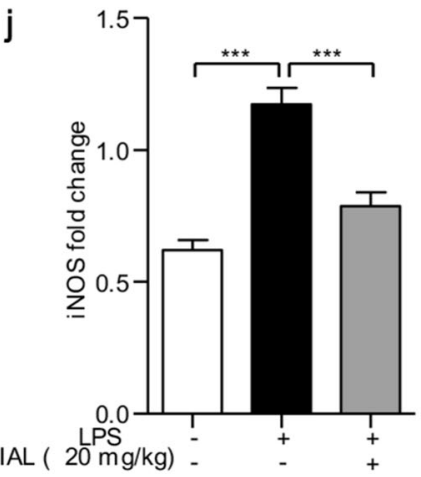

$L P S+I A L(20 \mathrm{mg} / \mathrm{kg})$

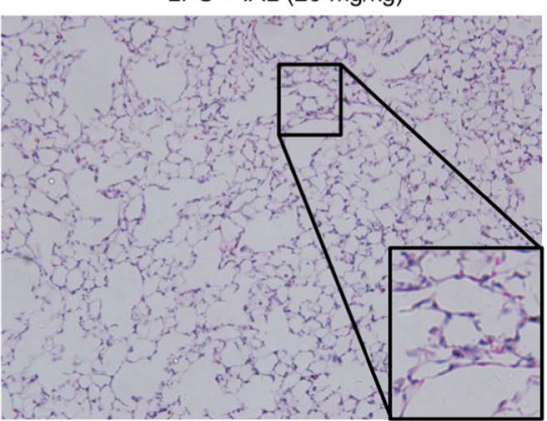

e

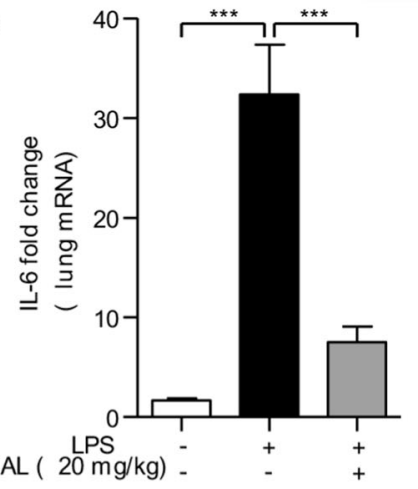

h
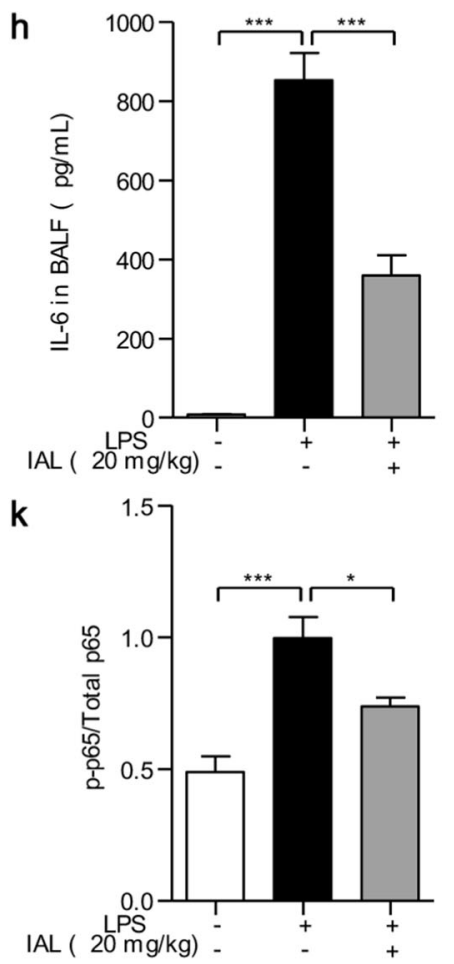

Fig. 5 IAL ameliorates pathological changes and pro-inflammatory cytokines in LPS-induced acute lung injury in vivo. a Brief procedure of the experiment. b H\&E staining of paraffin-embedded lung tissues from control, LPS alone-treated, and IAL-treated mice. Representative images of lung tissues in different groups were shown at the original magnification $(\times 200)$ and with partially enlarged details. c-e RT-PCR was performed to evaluate the mRNA expression changes of TNF- $\alpha$, IL-1 $\beta$, and IL- 6 in lung tissues. $\mathbf{f}-\mathbf{h}$ ELISA was performed to determine protein production in BALF. i The protein expression levels of iNOS, p-p65, and p65 were evaluated by western blotting. j,k Statistical analysis of iNOS expression and ratio of $\mathrm{p}$-p65/p65 in diagrams. The data represent the mean \pm SEM, $n=5$ mice for each group ${ }^{*} P<0.05$ and ${ }^{* * *} P<0.001$ ) 
a
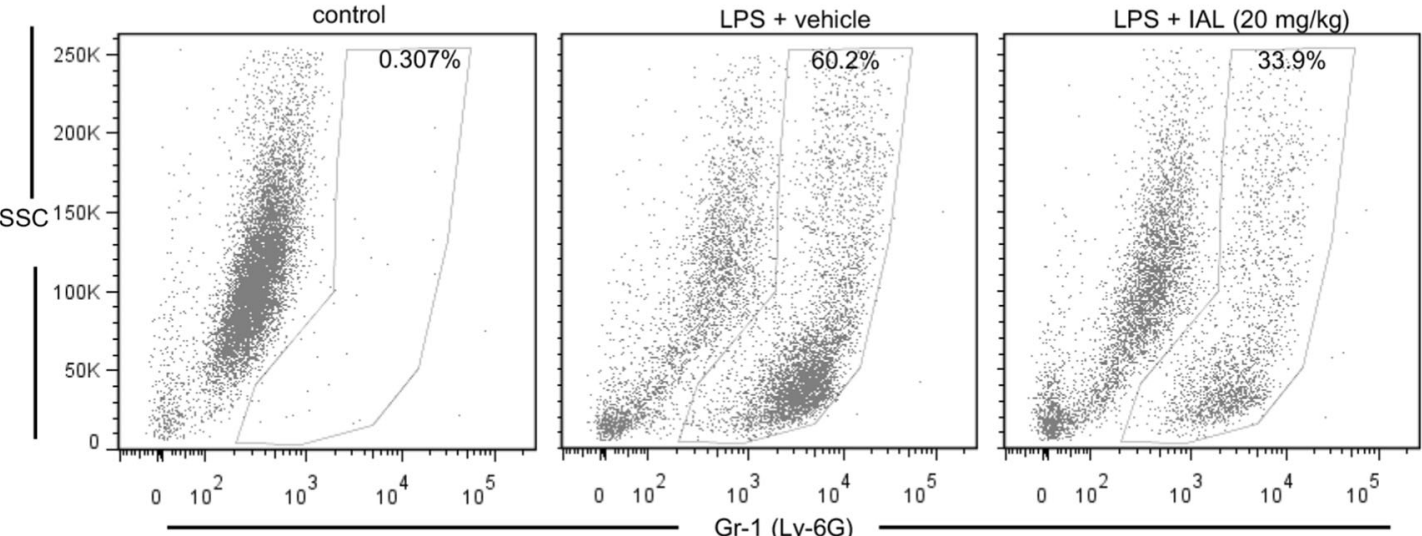

b

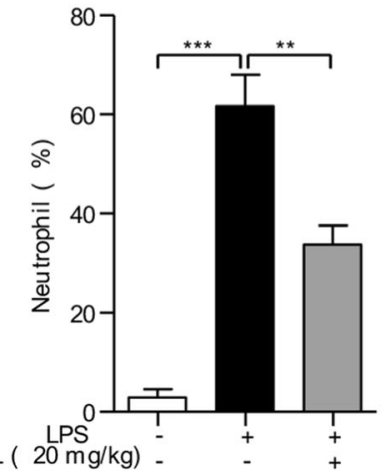

C

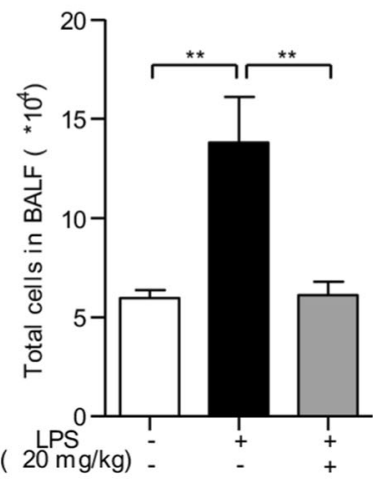

d

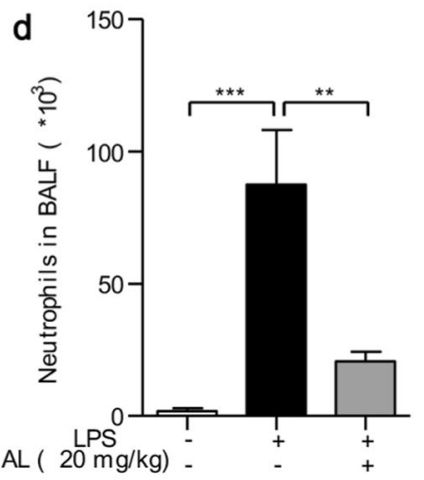

e
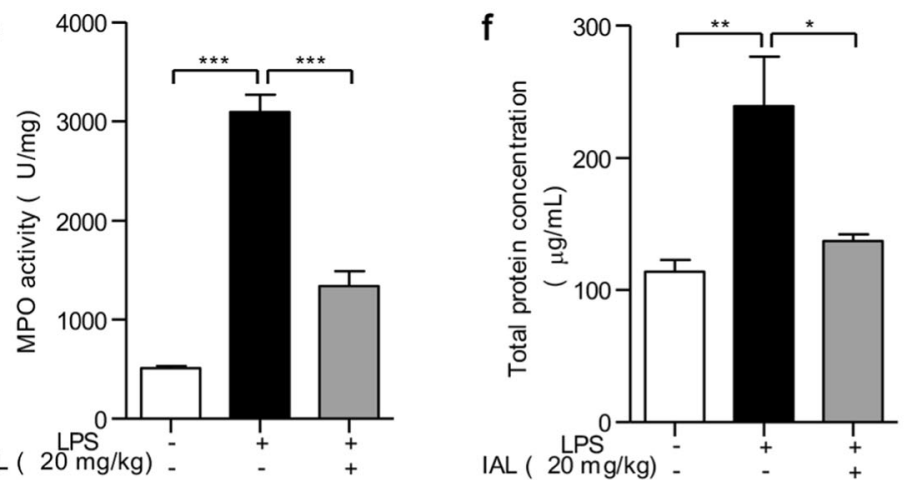

Fig. 6 IAL attenuates neutrophil infiltration, inflammatory cell accumulation, and pulmonary permeability in ALI mice. a Flow cytometry analysis of the neutrophil percentage in BALF. The FITC-positive cells were neutrophils. Statistical analysis of the neutrophil percentage is shown in b. c,d BALF was collected to measure the numbers of total cells and neutrophils. e The myeloperoxidase (MPO) activity in lung homogenates was determined. $\mathbf{f}$ The total protein concentration in BALF was detected. The data represent the mean \pm SEM, $n=5\left({ }^{*} P<0.05\right.$, ${ }^{*} P<0.01$, and $\left.{ }^{* * *} P<0.001\right)$

characteristic of transient immune activation in response to bacterial endotoxins [37]. Inhibition of these cytokines provides an important guidance for novel anti-inflammatory agents. In our study, IAL inhibited LPS-induced expression of pro-inflammatory cytokines in BMDMs at the mRNA and protein levels. These effects of IAL on macrophage activation suggest an anti-inflammatory role of IAL in bacteria-induced inflammation.

The inflammatory cytokines released during the inflammation process are reported to be regulated through the activation of MAPKs and the NF-KB signaling pathway [38]. To further investigate the anti-inflammation mechanism of IAL, we evaluated the expression and phosphorylation of MAPKs and NF-KB. Our results showed that IAL inhibited the phosphorylation of NF-KB p65, ERK, and AKT in LPS-stimulated BMDMs. However, the inhibitory effect of IAL on the phosphorylation of JNK and p38 MAPK was not significant. It was reported that IAL could overcome resistance to radiation in UMSCC-10A cells by specifically inhibiting the activation of the Erk1/2 signaling pathway [39]. The MAPKs are activated by phosphorylation caused by specific MAP kinase kinases (MKKs), which, in turn, are activated by MKK kinases [40]. In addition, the ERK pathway is associated with the regulation of cell growth and is activated by upstream MEK1 and MEK2 kinases, whereas the p38 pathway is activated by upstream MKK3 and MKK6, and the JNK pathway is activated by MKK4 and MKK7 [41]. Except for TRAF6, the upstream signals of MAPKs can also be regulated by small $G$ proteins. For instance, Ras can activate the ERK pathway while Rac/Cdc42 can activate p38 and JNK kinases $[40,41]$. TRAF6, a representative transcription factor, is activated by TLRs during bacterial or viral infection [42] and is responsible for the transcription of pro-inflammatory cytokine genes, which initiate an inflammatory cascade [43]. Modification of TRAF6 by means of ubiquitination is vital to the TLR-induced 


\section{a}
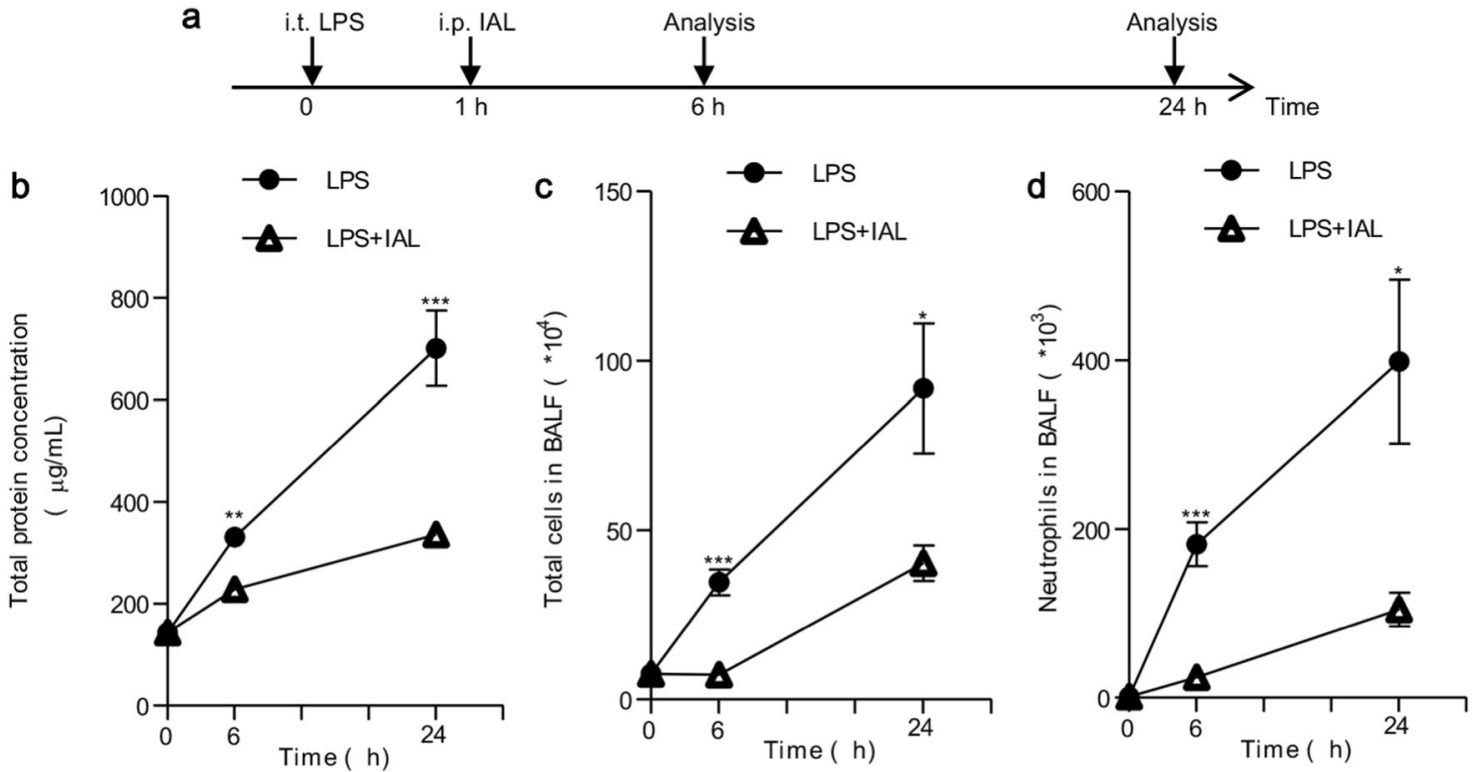

Fig. 7 IAL attenuates ALI with IAL treatment after LPS challenge. a Brief procedure of the experiment. $\mathbf{b}$ The total protein concentration in BALF was detected. $\mathbf{c}$ The numbers of total cells in BALF were measured. $\mathbf{d}$ The numbers of neutrophils in BALF were measured. The data represent the mean \pm SEM $\left(n=5 ;{ }^{*} P<0.05,{ }^{* *} P<0.01\right.$, and $\left.{ }^{* * *} P<0.001\right)$

inflammatory response [16]. The K48- and K63-linked polyubiquitin chains are the most common types of ubiquitin linkages [44]. K48-linked polyubiquitination has a role in proteasomal degradation, whereas K63-linked polyubiquitination has another role in cell signaling and serves as a scaffold to connect the upstream and downstream signaling proteins [45, 46]. TRAF6 contains a RING finger domain that is responsible for its E3 ubiquitin ligase activity of $\mathrm{K} 63$ polyubiquitination [47, 48], promoting the phosphorylation of TAK1 and activation of the IKK-NF-KB pathway [49]. Our results demonstrated that IAL restrains the TRAF6-dependent NF-KB activation using luciferase assay and non-degradable K63-linked ubiquitination of TRAF6 in HEK293 cells.

As macrophages are important inflammatory cells, they are also reported to promote neutrophil infiltration into the lungs and induce lung tissue damage [50]. ALI has caused a high mortality worldwide. However, no effective drugs have been reported thus far. During the pathogenesis of lung inflammatory disorders in ALI, various inflammatory mediators are released by endothelial cells, neutrophils, and macrophages into the alveolar space. Meanwhile, clinical studies indicate that a complex network comprising inflammatory cytokines and chemokines has a major role in the development of inflammatory diseases, including ALI [51]. These cytokines amplify lung inflammation in ALI patients, resulting in alveolar epithelial permeability and pulmonary edema [52]. The neutrophils infiltrate into the lung tissues, leading to the production of pro-inflammatory cytokines, which are secreted by activated alveolar macrophages in ALI/ARDS patients and influence pulmonary gas exchange $[2,53,54]$. In addition, due to the cytokines, the permeability of the alveolar-capillary barrier is increased, resulting in arterial hypoxemia, high protein pulmonary edema and respiratory failure $[55,56]$. Based on the effect of IAL on the activation of macrophages in vitro, we speculated that IAL can protect against ALI. LPS has been widely used in experimental ALI models because the intratracheal injection of LPS has been confirmed to imitate the pathophysiologic phenomenon observed in ALI patients [57]. The in vivo analysis performed in the ALI mouse model showed that IAL suppressed the release of pro-inflammatory cytokines, including TNF- $\alpha$, IL- $1 \beta$, and IL- 6 , in lung tissues and BALF. These results revealed that IAL might attenuate $L P S$-induced $A L I$ via inhibiting cytokine production in macrophages. Subsequently, we found that IAL attenuated the symptoms of LPS-induced ALI including neutrophil infiltration into the lung, pulmonary edema and histopathological changes. Both before and after LPS administration, IAL treatment ameliorated ALI and pulmonary inflammation, indicating that IAL have both protective and therapeutic roles in preventing and treating ALI.

In conclusion, the present study showed that IAL, a sesquiterpene lactone, could inhibit the production of inflammatory mediators in LPS-stimulated BMDMs. The inhibitory effect of IAL was partly attributed to the downregulation of non-degradable K63-linked ubiquitination of TRAF6, suppressing the activation of the NF-KB pathway. IAL could also attenuate inflammatory responses in the LPS-induced ALI mice model. Our results suggest that IAL might be a potential therapeutic anti-inflammatory reagent.

\section{ACKNOWLEDGEMENTS}

This study was supported by the National Natural Science Foundation of China [81773741, 81401302, 81573438, 31741038, and 81373424], the National Key Research and Development Program of China (2017YFC0908500) and Research Grant for Health Science and Technology of Pudong Municipal Commission of Health and family Planning of Shanghai (Grant Number PW2017A-24).

\section{AUTHOR CONTRIBUTIONS}

FQ and YD designed the study and drafted the manuscript. YS and YW participated in the project design and animal experiments. YD and TY performed the experiments. $\mathrm{HH}, \mathrm{YH}, \mathrm{DZ}, \mathrm{HJ}, \mathrm{KY}, \mathrm{XL}$, and LS helped in data analysis and discussion.

\section{ADDITIONAL INFORMATION}

Competing interests: The authors declare no competing interests.

\section{REFERENCES}

1. Grichnik KP, D'Amico TA. Acute lung injury and acute respiratory distress syndrome after pulmonary resection. Semin Cardiothorac Vasc Anesth. 2004;8:317-34.

2. Ware LB, Matthay MA. The acute respiratory distress syndrome. N Engl J Med. 2000;342:1334-49. 
3. Asimakopoulos G, Smith PL, Ratnatunga CP, Taylor KM. Lung injury and acute respiratory distress syndrome after cardiopulmonary bypass. Ann Thorac Surg. 1999;68:1107-15.

4. Grommes J, Soehnlein O. Contribution of neutrophils to acute lung injury. Mol Med. 2011;17:293-307.

5. Butt $Y$, Kurdowska A, Allen TC. Acute lung injury: a clinical and molecular review. Arch Pathol Lab Med. 2016;140:345-50.

6. Matthay MA, Ware LB, Zimmerman GA. The acute respiratory distress syndrome. J Clin Invest. 2012;122:2731-40.

7. Gando S, Kameue T, Matsuda N, Sawamura A, Hayakawa M, Kato H. Systemic inflammation and disseminated intravascular coagulation in early stage of ALI and ARDS: role of neutrophil and endothelial activation. Inflammation. 2004;28:237-44.

8. Chen T, Mou Y, Tan J, Wei L, Qiao Y, Wei T, et al. The protective effect of CDDO-Me on lipopolysaccharide-induced acute lung injury in mice. Int Immunopharmacol. 2015;25:55-64

9. Soler C, Valdes R, Garcia-Manteiga J, Xaus J, Comalada M, Casado FJ, et al. Lipopolysaccharide-induced apoptosis of macrophages determines the upregulation of concentrative nucleoside transporters Cnt1 and Cnt2 through tumor necrosis factor-alpha-dependent and -independent mechanisms. J Biol Chem. 2001;276:30043-9.

10. Yu LC, Flynn AN, Turner JR, Buret AG. SGLT-1-mediated glucose uptake protects intestinal epithelial cells against LPS-induced apoptosis and barrier defects: a novel cellular rescue mechanism? FASEB J. 2005;19:1822-35.

11. Hull C, McLean G, Wong F, Duriez PJ, Karsan A. Lipopolysaccharide signals an endothelial apoptosis pathway through TNF receptor-associated factor 6-mediated activation of C-Jun NH2-terminal kinase. J Immunol. 2002;169:2611-8.

12. Blackwell TS, Christman JW. The role of nuclear factor-kappa B in cytokine gene regulation. Am J Respir Cell Mol Biol. 1997;17:3-9.

13. Wang T, Hou W, Fu Z. Preventative effect of OMZ-SPT on lipopolysaccharideinduced acute lung injury and inflammation via nuclear factor-kappa B signaling in mice. Biochem Biophys Res Commun. 2017;485:284-9.

14. Lu YC, Yeh WC, Ohashi PS. LPS/TLR4 signal transduction pathway. Cytokine. 2008;42:145-51.

15. Chung JY, Park YC, Ye $\mathrm{H}$, Wu H. All TRAFs are not created equal: common and distinct molecular mechanisms of TRAF-mediated signal transduction. J Cell Sci. 2002;115:679-88.

16. Yu WW, Lu Z, Zhang H, Kang YH, Mao $Y$, Wang HH, et al. Anti-inflammatory and protective properties of daphnetin in endotoxin-induced lung injury. J Agric Food Chem. 2014:62:12315-25.

17. Beutler B. TIr4: central component of the sole mammalian LPS sensor. Curr Opin Immunol. 2000;12:20-6.

18. Zhou L, Ma Q, Shi H, Huo K. NUMBL interacts with TRAF6 and promotes the degradation of TRAF6. Biochem Biophys Res Commun. 2010;392:409-14.

19. Ma Q, Ruan H, Peng L, Zhang M, Gack MU, Yao WD. Proteasome-independent polyubiquitin linkage regulates synapse scaffolding, efficacy, and plasticity. Proc Natl Acad Sci USA. 2017;114:E8760-e9.

20. Chen ZJ, Sun L. Nonproteolytic functions of ubiquitin in cell signaling. Mol Cell. 2009:33:275-86

21. Wada T, Penninger JM. Mitogen-activated protein kinases in apoptosis regulation. Oncogene. 2004;23:2838-49.

22. Wu YX, He HQ, Nie YJ, Ding YH, Sun L, Qian F. Protostemonine effectively attenuates lipopolysaccharide-induced acute lung injury in mice. Acta Pharmacol Sin. 2018;39:85-96.

23. Medzhitov R, Horng T. Transcriptional control of the inflammatory response. Nat Rev Immunol. 2009;9:692-703.

24. Zhao K, Song X, Huang Y, Yao J, Zhou M, Li Z, et al. Wogonin inhibits LPS-induced tumor angiogenesis via suppressing PI3K/Akt/NF-kappaB signaling. Eur J Pharmacol. 2014;737:57-69.

25. Cragg GM, Newman DJ. Plants as a source of anti-cancer agents. J Ethnopharmacol. 2005;100:72-9.

26. Lajter I, Vasas A, Beni Z, Forgo P, Binder M, Bochkov V, et al. Sesquiterpenes from Neurolaena lobata and their antiproliferative and anti-inflammatory activities. $J$ Nat Prod. 2014;77:576-82.

27. Ciric A, Karioti A, Koukoulitsa C, Sokovic M, Skaltsa H. Sesquiterpene lactones from Centaurea zuccariniana and their antimicrobial activity. Chem Biodivers. 2012;9:2843-53.

28. Xiang P, Guo X, Han YY, Gao JM, Tang JJ. Cytotoxic and pro-apoptotic activities of sesquiterpene lactones from Inula britannica. Nat Prod Commun. 2016;11:7-10.

29. Di W, Khan M, Rasul A, Sun M, Sui Y, Zhong L, et al. Isoalantolactone inhibits constitutive NF-kappaB activation and induces reactive oxygen species-mediated apoptosis in osteosarcoma U2OS cells through mitochondrial dysfunction. Oncol Rep. 2014:32:1585-93.
30. He G, Zhang X, Chen Y, Chen J, Li L, Xie Y. Isoalantolactone inhibits LPS-induced inflammation via NF-kappaB inactivation in peritoneal macrophages and improves survival in sepsis. Biomed Pharmacother. 2017;90:598-607.

31. Chun J, Choi RJ, Khan S, Lee DS, Kim YC, Nam YJ, et al. Alantolactone suppresses inducible nitric oxide synthase and cyclooxygenase-2 expression by downregulating NF-kappaB, MAPK and AP-1 via the MyD88 signaling pathway in LPSactivated RAW 264.7 cells. Int Immunopharmacol. 2012;14:375-83.

32. Medzhitov R. Origin and physiological roles of inflammation. Nature. 2008;454:428-35.

33. Shenkar R, Abraham E. Mechanisms of lung neutrophil activation after hemorrhage or endotoxemia: roles of reactive oxygen intermediates, NF-kappa B, and cyclic AMP response element binding protein. J Immunol. 1999;163:954-62.

34. Wu J, Yan Z, Schwartz DE, Yu J, Malik AB, Hu G. Activation of NLRP3 inflammasome in alveolar macrophages contributes to mechanical stretch-induced lung inflammation and injury. J Immunol. 2013;190:3590-9.

35. Lee JH, Ko HJ, Woo ER, Lee SK, Moon BS, Lee CW, et al. Moracin M inhibits airway inflammation by interrupting the JNK/C-Jun and NF-kappaB pathways in vitro and in vivo. Eur J Pharmacol. 2016;783:64-72.

36. Korhonen R, Lahti A, Kankaanranta $\mathrm{H}$, Moilanen E. Nitric oxide production and signaling in inflammation. Curr Drug Targets Inflamm Allergy. 2005;4:471-9.

37. Wu Y, Jin F, Wang Y, Li F, Wang $L$, Wang $Q$, et al. In vitro and in vivo antiinflammatory effects of theaflavin-3,3'-digallate on lipopolysaccharide-induced inflammation. Eur J Pharmacol. 2017;794:52-60.

38. Kim KR, Son EW, Rhee DK, Pyo S. The immunomodulatory effects of the herbicide simazine on murine macrophage functions in vitro. Toxicol Vitr. 2002;16:517-23.

39. Fan $Y$, Weng Z, Gao H, Hu J, Wang H, Li L, et al. Isoalantolactone enhances the radiosensitivity of UMSCC-10A cells via specific inhibition of Erk1/2 phosphorylation. PLoS ONE. 2015;10:e0145790.

40. Zhang YL, Dong C. MAP kinases in immune responses. Cell Mol Immunol. 2005;2:20-7.

41. Dong C, Davis RJ, Flavell RA. MAP kinases in the immune response. Annu Rev Immunol. 2002;20:55-72.

42. Lawrence T. The nuclear factor NF-kappaB pathway in inflammation. Cold Spring Harb Perspect Biol. 2009;1:a001651.

43. Kim YY, Lee S, Kim MJ, Kang BC, Dhakal H, Choi YA, et al. Tyrosol attenuates lipopolysaccharide-induced acute lung injury by inhibiting the inflammatory response and maintaining the alveolar capillary barrier. Food Chem Toxicol. 2017;109:526-33.

44. Husnjak K, Dikic I. Ubiquitin-binding proteins: decoders of ubiquitin-mediated cellular functions. Annu Rev Biochem. 2012;81:291-322.

45. Zhang J, Zhang C, Cui J, Ou J, Han J, Qin Y, et al. TRIM45 functions as a tumor suppressor in the brain via its E3 ligase activity by stabilizing p53 through K63linked ubiquitination. Cell Death Dis. 2017;8:e2831.

46. Chen ZJ. Ubiquitin signalling in the NF-kappaB pathway. Nat Cell Biol. 2005;7:758-65

47. Chiu YH, Zhao M, Chen ZJ. Ubiquitin in NF-kappaB signaling. Chem Rev. 2009;109:1549-60.

48. Lee Y, Lee IY, Yun HJ, Lee WS, Kang S, Cho SG, et al. BAT3 negatively regulates lipopolysaccharide-induced NF-kappaB signaling through TRAF6. Biochem Biophys Res Commun. 2016;478:784-90.

49. Deng L, Wang C, Spencer E, Yang L, Braun A, You J, et al. Activation of the IkappaB kinase complex by TRAF6 requires a dimeric ubiquitin-conjugating enzyme complex and a unique polyubiquitin chain. Cell . 2000;103:351-61.

50. Gauna $A E, C h a S$. Akt2 deficiency as a therapeutic strategy protects against acute lung injury. Immunotherapy. 2014;6:377-80.

51. Goodman RB, Pugin J, Lee JS, Matthay MA. Cytokine-mediated inflammation in acute lung injury. Cytokine Growth Factor Rev. 2003;14:523-35.

52. Ganter MT, Roux J, Miyazawa B, Howard M, Frank JA, Su G, et al. Interleukin-1 beta causes acute lung injury via alphavbeta5 and alphavbeta6 integrin-dependent mechanisms. Circ Res. 2008;102:804-12.

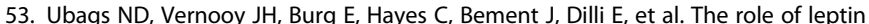
in the development of pulmonary neutrophilia in infection and acute lung injury. Crit Care Med. 2014;42:e143-51.

54. Parsey MV, Tuder RM, Abraham E. Neutrophils are major contributors to intraparenchymal lung IL-1 beta expression after hemorrhage and endotoxemia. J Immunol. 1998;160:1007-13.

55. Lewis JF, Jobe AH. Surfactant and the adult respiratory distress syndrome. Am Rev Respir Dis. 1993;147:218-33.

56. Ware LB. Pathophysiology of acute lung injury and the acute respiratory distress syndrome. Semin Respir Crit Care Med. 2006;27:337-49.

57. Chen $H$, Bai $C$, Wang $X$. The value of the lipopolysaccharide-induced acute lung injury model in respiratory medicine. Expert Rev Respir Med. 2010;4:773-83. 